\title{
Day case laparoscopic radical prostatectomy
}

\author{
Hamid Abboudi ${ }^{1}$, Patrick Doyle ${ }^{2}$, Mathias Winkler ${ }^{1}$ \\ ${ }^{1}$ Departments of Urology and ${ }^{2}$ Anaesthesia, Charing Cross Hospital, Imperial College Healthcare NHS Trust, London, UK.
}

\begin{abstract}
Summary Background: To evaluate the feasibility of performing laparoscopic radical prostatectomy (LRP) as a day case procedure while maintaining patient satisfaction and safety. Herein we report our experience, selection criteria, and discharge criteria for day case LRP.

Methods: We performed a prospective study with 32 patients undergoing extraperitoneal LRP. These patients were counselled before the procedure that they would go home the same evening of the procedure. Pain scores and quality of life data were recorded day 1 postoperatively via a telephone consultation. The patients underwent routine blood tests on day 2 and an outpatient review on day 7 and regularly thereafter via an assigned key worker. Socio-demographic data, comorbidities, and outcomes were collected for analysis.

Results: All patients were successfully discharged the same day of surgery. Mean patient age was 62 years with a mean body mass index of 25. Mean operative time was 147 minutes, and estimated blood loss was $101 \mathrm{ml}$. Three patients were treated for post operative urinary tract infections; two patients developed infected lymphoceles which required percutaneous drainage and one patient required re-catheterisation due to a burst catheter balloon. Of these six complications four patients required re-admission. Post-operative pain, nausea and vomiting were low whilst patient satisfaction scores were unanimously high in all patients surveyed.

Conclusions: The early experience with extraperitoneal LRP as a same day surgery is promising although patients who are at high risk of lymphocele should be excluded.

Preoperative patient counselling and selection is paramount. Patient satisfaction is not adversely affected by the shortened stay. Surgeon experience, a well-motivated patient, meticulous attention to detail through an integrated pathway, a multidisciplinary team and adequate postoperative assessment are essential.
\end{abstract}

KEY WORDS: Day surgery; Laparoscopy; Laparoscopy malignant disease; Prostate cancer.

Submitted 16 May 2017; Accepted 2 July 2017

\section{INTRODUCTION}

Since Schuessler first described the technique over 20 years ago (1), laparoscopic radical prostatectomy (LRP) has been established as an effective minimally invasive surgical treatment option for localised prostate cancer with the advantages of decreased blood loss, decreased analgesic requirements and earlier hospital discharge and convalescence compared with open radical prostatectomy (2-6). Hospital stays have shortened dramatically over the years such that it has become standard practice for patients to spend 1-2 nights in hospital after minimally invasive radical prostatectomy with benefits to both patients and healthcare providers. These incentives for early discharge, however, must always be trumped by the patient's best interest i.e. safety and in particular readmission rates.

In 2012 we reported the evolution of a care pathway for LRP that set out to systematically reduce the impact of surgery to the patient through thorough patient preparation and complete minimization of perioperative symptoms (7). Our aim was to evaluate the feasibility of performing LRP as a day case procedure while maintaining patient satisfaction and safety.

Herein we report our experience and to our knowledge the first in the published literature the selection and discharge criteria and outcomes for day case LRP.

\section{Patients And methods}

Patients were considered for same day discharge if they met the following conditions: ASA 1 or 2 , BMI $<30$, short procedure with minimal blood loss, minimal perioperative opioids, watertight anastomosis, minimal discomfort or malaise, drain not required and responsible adult at home. Demographics, perioperative and follow up data were prospectively collected and recorded on a database.

Patients received a standardized general anaesthetic with fentanyl as the opioid of choice intra-operatively and transversus abdominis plane (TAP) blocks with bupivacaine $0.5 \%$ were routinely performed for all patients (Figure 1). Towards the end of surgery they received paracetamol and a non-steroidal anti-inflammatory drug. Invasive monitoring was not utilized. Post-operative pain in the initial recovery period was managed with morphine sulfate as required. Once on the ward, patients received regular paracetamol, diclofenac and oxynorm as required.

All of the patients were scheduled as the first case of the day to allow for clinical assessment by the consultant urologist before discharge in the evening of surgery.

All cases were performed by an experienced surgeon who had performed > 100 LRPs using the technique described by Stolzenburg which utilizes extraperitoneal balloon dissection (8). Extra attention was given to careful haemostasis which included the use of intracorporeal haemostatic adjuncts such as Surgiflo ${ }^{T M}$ and Surgicel ${ }^{\mathrm{TM}}$. 
Figure 1.

Transverse ultrasound view of classic 'crescent shape' of the fascial layers splitting between IOAM and TAM on injection of local anaesthetic.

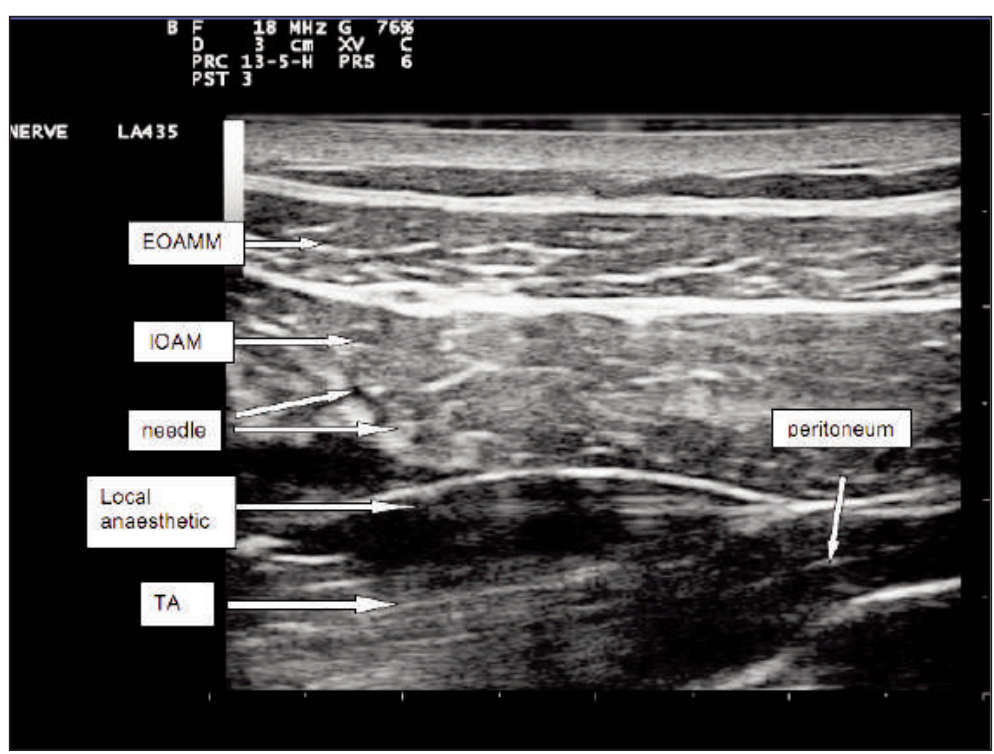

The median time spent in hospital was 12 hours (7am to $7 \mathrm{pm}$ ) and the latest discharge time was $8 \mathrm{pm}$.

Mean total operative time (i.e. from skin to skin) was 147 minutes. The mean estimated blood loss was $101 \mathrm{~mL}$. Seven patients had bilateral pelvic lymph node dissection. There were no significant perioperative complications in this series of patients. In total six patients developed postoperative complications of which 4 required re-admission (Table 2). Three patients were treated for post-operative urinary tract infections; two patients developed infected lymphoceles which required percutaneous drainage and one patient required re-catheterization due to a burst catheter balloon (equipment failure). No events requiring general anaesthetic were observed (Clavien 3b).

Sixteen of the 32 patients agreed to participate in the survey portion of the study. The other sixteen patients were not surveyed on day 1 postoperatively, but did not have any immediate issues on review of their medical records.

Generally the use of drains was avoided. A leak test with $250 \mathrm{cc}$ of normal saline was always performed to guarantee a strong and watertight anastomosis and avoid anastomotic leak. No special restrictions were placed on resident or fellow participation, which received modular training as customary in teaching hospitals.

Post operatively patients were allowed to eat and drink as soon as they wished and early mobilization was encouraged. Patients were provided with direct telephone contact details of the surgeon and specialist nurse in case of any early complications not recognized prior to discharge (Appendix A).

Patients were followed up with a telephone consultation and questionnaire on the first day post-operatively to address safety concerns and accurate prospective capturing of complications. Patients were specifically asked to score their level of post-operative pain and nausea and vomiting and satisfaction on a linear scale from 1-5 with 1 being no pain/very satisfied and 5 representing dissatisfaction and worst pain imaginable (Appendix A). Nurse led trial without catheter took place seven days post operatively where the catheter was removed without prior cystogram. Routine catheter urine and midstream urine cultures were obtained to seek out proactively and treat infections at the time of catheter removal and again at first outpatient review. Outpatient review was arranged early at 30 days for the same reason. All complications were classified according to the modified Clavien-Dindo system.

\section{RESULTS}

Patient demographics, relevant pre-operative clinical and pathological data are illustrated (Table 1).

Between June 2009 and December 2014, 353 laparoscopic radical prostatectomies were performed. Of these 32 were deemed suitable for same day discharge. In total all 32 patients were discharged on the same day.
All patients were discharged from hospital in the evening of their surgery without complication.

Table 1.

Demographic data and salient descriptive statistics of day case prostatectomy patients.

\begin{tabular}{|l|c|c|}
\hline & & Day case patients $(\mathbf{n}=\mathbf{3 2})$ \\
\hline Age (years) & & 62 \\
\hline Pre-op PSA (ng/ml) & & 8.58 \\
\hline Clinical stage (\%) & T1c & 56 \\
& T2 a/b & 31 \\
T2c & 13 \\
\hline Pre op Gleason sum (\%) & $3+3=6$ & 41 \\
& $3+4=7$ & 50 \\
& $4+3=7$ & 3 \\
\hline Operating time (min) & $4+5=9$ & 6 \\
\hline Blood loss (ml) & & 147 \\
\hline Transfusion rate & & 101 \\
\hline Conversion & & 0 \\
\hline LoS (days) & & 0 \\
\hline
\end{tabular}

Table 2.

Clavien-Dindo classification of complications for cohort.

\begin{tabular}{|l|c|c|}
\hline & & Day case patients $(\mathbf{n}=\mathbf{3 2})$ \\
\hline ASA & Class 1 & 12 \\
& Class 2 & 20 \\
\hline Clavien & None & 0 \\
& 1 & 0 \\
& 2 & 3 \\
& $3 a$ & 3 \\
& $3 b$ & 0 \\
& $4 a$ & 0 \\
& $4 b$ & 0 \\
& 5 & 0 \\
\hline
\end{tabular}


Table 3.

Functional and oncological outcomes.

\begin{tabular}{|lc|lc|}
\hline Continence & $\begin{array}{c}\text { Number } \\
\text { of patients }\end{array}$ & $\begin{array}{l}\text { Erectile } \\
\text { dysfunction }\end{array}$ & $\begin{array}{c}\text { Number } \\
\text { of patients }\end{array}$ \\
\hline Dry & 27 & Potent & 14 \\
\hline $1-2$ pads/day & 4 & Mild ED & 9 \\
\hline 3 or more pads/day & 1 & No erections & 4 \\
\hline Total & 32 & Not interested in erections & 5 \\
\hline
\end{tabular}

All patients reported post-operative satisfaction scores of 1 (n-14) or 2 (n-2) out of 5. Post-operative pain was deemed minimal in 14 out of 16 , with one patient scoring their pain 3 out of 5 and one patient scoring their pain 4 out of 5 . Similarly day 1 nausea and vomiting was absent in all patients.

Functional outcomes were assessed at 3 months post LRP (Table 3). Twenty-seven patients were dry, whilst four patients were using 1-2 pads per day, and one patient required 3 or more pads per day. In terms of erectile dysfunction (ED) 14 patients were completely potent, nine patients had partial ED, four patients could not achieve erections and five patients were not interested in sexual activity. At 46 months median follow up 11 of the 32 patients showed evidence of biochemical recurrence, with no prostate cancer related deaths in the series. All eleven patients went on to have salvage radiotherapy.

\section{Discussion}

There continues to be growing concern over the cost of national health services in the United Kingdom.

Resources are limited and our aging population continues to expand. Several studies have looked at the potential economic effect of laparoscopic versus open versus robotic surgery prostate cancer. Robotic surgery adds significant additional costs to the hospital, with several studies showing cost advantages with laparoscopic over robotic prostate surgery when excluding case volume.

Our aim was to investigate whether the procedure could be performed as a same day surgical procedure, which to our knowledge has not been reported with respect to LRP. One night in hospital amounts to approximately $£ 700$ a night thus such a pathway amounts to a substantial saving if implemented safely. Further theoretical cost advantages to the hospital include a greater reimbursement tariff for day case procedures over in-patient stay as well as the cost savings with regards to a hospital bed saving. With regards to the United Kingdoms National Health Service, the aim should be to perform more day case prostatectomies in order to negotiate higher tariffs with the clinical commissioning groups so that hospitals could be reimbursed for an 'outpatient prostatectomy' and ultimately decrease overall costs. The authors recognize that this may not be applicable to other healthcare systems, however the benefits of reduced time in hospital such as early mobility, reduced infection risk and psychological benefits of being at home are transferrable to the wider healthcare community. The incentives for early discharge, however, must always be trumped by the patient's best interest and thus there is a need to maintain a low threshold for admitting a patient following LRP. Consequently careful case selection and a review by the senior operating surgeon prior to discharge should be mandatory.

Utilizing the extra-peritoneal approach minimizes the chances of post-operative ileus and other bowel complications and has the potential to reduce time in hospital. This was also shown with the robotically assisted approach in a small patient cohort (9). Patients with a BMI > 30 are more likely to have surgical complications and pose challenges to the anaesthetist and surgeon alike. Such patients lead to a more technically challenging dissection with narrowing of the operative field within the extraperitoneal space. We therefore opted to admit such patients for observation.

We found that patients could be discharged home the same day of the surgical procedure if they met strict criteria that we set without increased perioperative complications. We also performed follow up satisfaction surveys and found that all patients who completed the survey were satisfied with their experience and that they stayed for the appropriate amount of time.

The observed complications are in line with contemporary series except a high rate of infected lymphoceles in 2 of 7 patients with pelvic lymphadenectomy. This is reflected in a high readmission rate of $12 \%$. We conclude that it is best to insert a post-operative drain for a minimum of 24 hours after extended lymphadenectomy and patients be given the choice of being discharged with the drain in situ or being admitted overnight for observation. We also acknowledge that in order to increase the yield of day case surgery candidates, including selected patients who have a drain left in situ as potential day case candidates would be safe. Discharging patients with drains postoperatively is routine practice in some surgical disciplines and therefore should not preclude LRP patients from similar benefits.

The success of this technique is in the standardized, team approach in the context of a well-motivated medically fit patient. It was realized early that the majority of pain arises from the anterior abdominal wall after extraperitoneal LRP. As such, minimizing post-operative pain through a transabdominal plane (TAP) block within the pathway and sparing use of opioids to overcome postoperative nausea and vomiting have proved effective (7). The majority of patients only needed a combination of Paracetamol and NSIADs for post-operative pain. The probability of side effects from opioids for break-through pain was minimized by switching from Tramadol (partial agonist and antagonist) to Oxynorm, which has an improved side effect profile.

We report the outcomes of a pilot cohort of patients, without a control group to compare to. We aim to conduct studies of suitable patients to further validate the findings reported. The percentage of patients surveyed was also low and thus any further studies should aim to have a higher participation from included patients. Furthermore we utilized an unvalidated questionnaire tool that was constructed in house by the research team. In future validated surveys such as the Patient Judgment 
System-24 (PJS-24) questionnaire should be utilized. The PJS-24 gives a global overall satisfaction with care rating and 9 multi-item satisfaction components.

The PJS-24 has been validated for hospital quality assurance and shown to accurately reflect and capture issues of patients undergoing prostatectomy $(10,11)$. A cost analysis compared to the standard patient pathway would be a welcome addition to the literature and would provide further evidence for the feasibility of such a pathway.

\section{Conclusion}

The early experience with extraperitoneal LRP with same day discharge home is promising.

Preoperative patient counselling and selection is paramount. Patient satisfaction is not adversely affected by the shortened stay. Surgeon experience, a well-motivated patient, meticulous attention to detail through an integrated pathway, a multidisciplinary team and adequate postoperative assessment are essential.

\section{REFERENCES}

1. Schuessler W, Schulam P, Clayman R, Kavoussi L. Laparoscopic radical prostatectomy: initial short-term experience. Urology. 1997; 50:854-7.

2. Eden CG, Neill MG, Louie-Johnsun MW. The first 1000 cases of laparoscopic radical prostatectomy in the UK: evidence of multiple "learning curves". BJU Int. 2009; 103:1224-30.

3. Rassweiler J, Stolzenburg J, Sulser T, et al. Laparoscopic radical prostatectomy-the experience of the German Laparoscopic Working Group. Eur Urol. 2006; 49: 113-19.

4. Stolzenburg J, Kallidonis P, Minh D, et al. Endoscopic extraperitoneal radical prostatectomy: evolution of the technique and experience with 2400 cases. J Endourol. 2009; 23:1467-72

5. Touijer K, Eastham J, Secin FP, Romero Otero J, et al. Comprehensive prospective comparative analysis of outcomes between open and laparoscopic radical prostatectomy conducted in 2003 to 2005. J Urol. 2008; 179:1811-17.

6. Bhayani SB, Pavlovich CP, Hsu TS, et al. Prospective comparison of short-term convalescence: laparoscopic radical prostatectomy versus open radical retropubic prostatectomy. Urology. 2003; 61:612-16.

7. Dudderidge T, Doyle P, Mayer E, et al. Evolution of care pathway for laparoscopic radical prostatectomy. J Endourol. 2012; 26:660-665.

8. Stolzenburg J, Kallidonis P, Minh D, et al. Endoscopic extraperitoneal radical prostatectomy: evolution of the technique and experience with 2400 cases. J Endourol. 2009; 23:1467-1472.

9. Martin A, Nunez R, Andrews J, et al. Outpatient prostatectomy: too much too soon or just what the patient ordered. Urology. 2010; 75:421-424.

10. Litwin MS, Shpall AI, Dorey F. Patient satisfaction with short stays for radical prostatectomy. Urology. 1997; 49:898-906.

11. Hays $R D$, Larson C, Nelson E, Batalden P. Hospital quality trends: a short-form patient-based measure. Med Care. 1991; 29:661-668.

\section{Correspondence}

Hamid Abboudi, MD

Mathias Winkler, MD

mathias.winkler@imperial.nhs.uk

Department of Urology, Charing Cross Hospital,

Imperial College Healthcare NHS Trust London (UK)

Patrick Doyle, MD

Department of Anaesthesia, Charing Cross Hospital, Imperial College

Healthcare NHS Trust - London (UK) 